Research Article

\title{
Synthesis and Antioxidative Activity of Piperine Derivatives Containing Phenolic Hydroxyl
}

\author{
Bei Qin $\mathbb{D}^{1,2}$ Kuan Yang, ${ }^{2,3}$ and Ruijun Cao ${ }^{1}$ \\ ${ }^{1}$ School of Science, Xi'an Jiaotong University, Xi'an 710049, Shaanxi, China \\ ${ }^{2}$ Department of Pharmacy, Xi'an Medical University, Xi'an 710021, Shaanxi, China \\ ${ }^{3}$ College of Bioresources Chemical and Materials Engineering, Shaanxi University of Science and Technology, Xi'an 710021, \\ Shaanxi, China \\ Correspondence should be addressed to Bei Qin; qinbei0526@163.com
}

Received 15 April 2020; Revised 16 June 2020; Accepted 23 June 2020; Published 21 July 2020

Academic Editor: Ioannis G. Roussis

Copyright (c) 2020 Bei Qin et al. This is an open access article distributed under the Creative Commons Attribution License, which permits unrestricted use, distribution, and reproduction in any medium, provided the original work is properly cited.

\begin{abstract}
Piperine was used in this study in its raw form, and different steps, such as amide hydrolysis and amidation, were used to synthesize piperine derivatives containing a phenolic hydroxyl group. DPPH and ABTS free radical scavenging assays were used to assess piperine derivative antioxidant activities. We constructed an AAPH oxidative stress erythrocyte model to study the effect of piperine derivatives on the hemolysis rate of oxidatively damaged erythrocytes as well as the hemoglobin oxidation rate. This AAPH model was also used to determine piperine derivative effects on antioxidant enzyme activity and malondialdehyde (MDA) content. Results showed that spectroscopic methods could synthesize and identify piperine derivatives containing phenolic hydroxyl groups $(\mathrm{H}-1 \sim \mathrm{H}-3)$. Moreover, $\mathrm{DPPH}$ and ABTS assay results showed that piperine derivative free radical clearance rates were higher compared with the parent compound. Additionally, piperine derivatives $(\mathrm{H}-1 \sim \mathrm{H}-3)$ were found to provide protection to AAPH oxidatively damaged erythrocytes in their ability to inhibit AAPH-induced erythrocyte lysis, while hemoglobin oxidation was higher compared with the parent compound. Piperine derivatives may protect intracellular glutathione peroxidase (GSH-Px) antioxidant enzyme system activities, safeguarding against oxidative damage. This study synthesized novel piperine derivatives for use as potential antioxidant agent candidates.
\end{abstract}

\section{Introduction}

Free radicals refer to any molecule with one or more unpaired electrons that is a product of normal body metabolism [1]. Some free radicals participate in cellular activities in a controlled manner, while others are unbounded and interact with nucleic acids, lipids, proteins, and other important biological molecules [2], causing acute and chronic dysfunctions to occur. Accordingly, it is widely believed that free radicals, which induce oxidative stress, are the main contributors in the occurrence and development of multiple diseases, fueling mechanisms that lead to cerebrocardiovascular disease (CVD), tumors, inflammation, and neurodegenerative diseases [2-4]. However, antioxidants can scavenge excess free radicals within the body. As a result, the discovery of novel antioxidant drugs has attracted scientific interest and has led to much research $[5,6]$.
Piperine is an active alkaloid that is extracted from peppers, long peppers, and other plants. It produces a variety of physiological and pharmacological effects to occur, such as antioxidative [7], antilipid [8], immunoregulative, antitumor [9], and anti-inflammatory effects [10]. One study found that piperine inhibited oxidative damage levels that could potentially be associated with its effect on reducing free radicals and reactive oxygen species (ROS) [7]. The antioxidative effects of piperine are derived by the inhibition of lipid peroxidation and the enhancement of glutathione (GSH) synthesis or transport [11]. Studies have shown that piperine antioxidant activities are associated with the molecule's carbon-oxygen five-membered rings and amide structure. Piperine reportedly undergoes demethylation of its carbon-oxygen five-membered rings during metabolism in the human body, mainly producing metabolites with a bisphenol hydroxyl structure [12]. The consequent synthesis 
of phenolic hydroxyl groups enhances piperine antioxidant activity [13]; hence, this enhancement should effectually be retained during structural modification. Several studies have shown that the derivatization of amide components within piperine could also improve its antioxidant activity capacity [14]. For example, Kharbanda et al. [15] derivatized the amide structure of piperine to synthesize a series of piperic acid and benzothiazole conjugates, and they found that the derivatives significantly reduced factors associated with PPAY $\gamma$ activity, consequently regulating lipid peroxidation [15]. Several studies on natural antioxidants have reported that most antioxidants were polyphenols, except for glycosides and proteins, indicating that the presence of phenolic hydroxyl groups effectively improves the reducing power of compounds. Accordingly, this study derivatized the amide structure of piperine to introduce phenolic hydroxyl groups, consequently obtaining phenolic hydroxylcontaining piperine derivatives with improved antioxidant capacities.

\section{Materials and Methods}

2.1. Chemicals and Experimental Animals. Oxalyl chloride (98\%) was purchased from Saan Chemical Technology Co., Ltd (Shanghai, China). TCI Development Co., Ltd (Shanghai, China), supplied 3-aminophenol (98.5\%). J\&K Scientific Co., Ltd, supplied 4-aminophenol (97\%) and 2aminophenol (99\%). Piperine was purchased from Shaanxi Ciyuan Biotechnology Co., Ltd. Dichloromethane (DCM), petroleum ether (PE), methanol, and ethyl acetate (EA; analytical grade) were all purchased from Tianjin Fuyu Fine Chemical Co., Ltd. The methemoglobin $(\mathrm{Hb})$ reagent kit, catalase (CAT) visible light reagent kit, total protein quantitation (BCA assay) reagent kit, malondialdehyde (MDA) reagent kit, glutathione peroxidase (GSH-Px) reagent kit, and total superoxide dismutase (T-SOD) reagent kit were all purchased from the Nanjing Jiancheng Bioengineering Institute. AAPH (2, 2'-azobis (2-amidinopropane) dihydrochloride) was purchased from Saan Chemical Technology Co., Ltd.

Male Sprague Dawley laboratory rats $(280 \pm 10 \mathrm{~g})$ were obtained from Chengdu Dossy Experimental Animals Co., Ltd. All experimental animals underwent routine feeding and housing as well as ad libitum access to food and water. The experimental procedures used in this study were approved by the Ethics Committee of Xi'an Medical University.

2.2. Piperine Derivative Synthesis. Piperine was used as a raw material to synthesize three structurally modified piperine derivatives based on the pathways shown in Figure 1.

2.2.1. (2E, 4E)-5-(Benzo[d][1,3]dioxol-5-yl)penta-2,4-dienoic $\operatorname{Acid}\left(1, \mathrm{C}_{12} \mathrm{H}_{10} \mathrm{O}_{4}\right)$. Piperine $(10.69 \mathrm{~g}, 37.51 \mathrm{mmol})$ was dispersed in a potassium hydroxide $(\mathrm{KOH})(237 \mathrm{~g}$, $4.22 \mathrm{~mol}) /$ methanol solution $(300 \mathrm{~mL})$ and refluxed at $75^{\circ} \mathrm{C}$. After $24 \mathrm{~h}$, the solution was cooled and subjected to suction filtration to obtain a white solid. The solid was dispersed in small amounts of methanol, and $6 \mathrm{~mol} / \mathrm{L}$ hydrochloric acid was used to adjust the $\mathrm{pH}$ level to one. Following this, suction filtration was conducted, after which the substance was dried to obtain compound 2 . The product was a yellow powder with a mass of $7.61 \mathrm{~g}$ and a yield of $93.1 \% .{ }^{1} \mathrm{H}$ NMR $\left(400 \mathrm{MHz}, \mathrm{DMSO}-\mathrm{d}_{6}\right): \delta 12.21(\mathrm{~s}, 1 \mathrm{H},-\mathrm{COOH}), 7.30$ (ddd, $J=15.3,7.0,3.4 \mathrm{~Hz}, 1 \mathrm{H}, \mathrm{CH}=\mathrm{CH}-\mathrm{CO}), 7.24(\mathrm{~d}, J=1.7 \mathrm{~Hz}$, 1H, Ar-H), 7.03-6.79 (m, 3H, Ar-H, Ar- $\mathrm{CH}=\mathrm{CH}-), 6.93$ (d, $J=8.0 \mathrm{~Hz}, 1 \mathrm{H}, \mathrm{Ar}-\mathrm{CH}=\mathrm{CH}-), 6.06\left(\mathrm{~s}, 2 \mathrm{H},-\mathrm{OCH}_{2} \mathrm{O}-\right), 5.93$ (d, $J=15.2 \mathrm{~Hz}, 1 \mathrm{H}, \mathrm{CH}=\mathrm{CH}-\mathrm{CO}) .{ }^{13} \mathrm{C}$ NMR $(101 \mathrm{MHz}$, DMSO- $\mathrm{d}_{6}$ ): $\delta 168.03,148.56,148.45,145.01,140.21,131.00$, $125.33,123.51,121.63,108.97,106.20,101.82$.

2.2.2. (2E, 4E)-5-(Benzo[d][1,3]dioxol-5-yl)penta-2,4-dienoyl Chloride $\left(2, \mathrm{C}_{12} \mathrm{H}_{9} \mathrm{ClO}_{3}\right)$. Compound $2(6.91 \mathrm{~g}, 31.7 \mathrm{mmol})$ was dispersed in $15 \mathrm{~mL}$ of anhydrous DCM. An oxalyl chloride-DCM solution $(12 \mathrm{~mol} / \mathrm{L}, 2.70 \mathrm{~mL})$ was added and stirred for $2 \mathrm{~h}$ at room temperature to obtain an orange liquid. Vacuum distillation was used to remove oxalyl chloride and DCM to obtain an orange-red amide. It is important to note that this amide must be prepared fresh before use.

2.2.3. Synthesis of Piperine Derivatives Containing Phenolic Hydroxyl Groups (H-1, H-2, and H-3). As shown in Figure 1, the reaction solution (4-aminophenol (2-aminophenol, 3aminophenol; $50.2 \mathrm{mg}, 0.46 \mathrm{mmol}$ )) was added to a $25 \mathrm{~mL}$ round-bottom flask along with $2 \mathrm{~mL}$ of DCM. After $5 \mathrm{~min}$ of ultrasonic dispersion, a newly prepared saturated sodium bicarbonate $\left(\mathrm{NaHCO}_{3}\right)$ aqueous solution $(0.4 \mathrm{~mL})$ was added, followed by the slow addition of a freshly prepared piperonyloyl chloride-DCM solution $(0.5 \mathrm{mmol})$. Next, $2 \mathrm{~mL}$ of DMF was added to clarify the reaction system. This was followed by stirring at room temperature for 3-4 h. After thin-layer chromatography detected the 4 -aminophenol (2aminophenol, 3-aminophenol), $10 \mathrm{~mL}$ of distilled water was added under stirring action to quench the reaction. Following this, $15 \mathrm{~mL}$ of EA was added before washing with a $5 \% \mathrm{NaHCO}_{3}$ aqueous solution $(2 \times 20 \mathrm{~mL}), 5 \% \mathrm{HCl}$ $(2 \times 20 \mathrm{~mL})$, and distilled water $(2 \times 20 \mathrm{~mL})$. The solution was washed, concentrated, and vacuum-dried to obtain a paleyellow crude product.

The H-1 crude product was then purified using column chromatography on a silica gel (mixture eluent: $\mathrm{EA}: \mathrm{PE}=2: 1$ ) to obtain an $84 \mathrm{mg}$ product (yield: $58 \%$ ) as a pale-yellow solid. ${ }^{1} \mathrm{H}$ NMR (400 MHz, DMSO-d $\left.\mathrm{d}_{6}\right): \delta 9.89$ (s, $\left.1 \mathrm{H},-\mathrm{CO}-\mathrm{NH}-\right)$, $7.47(\mathrm{~d}, J=8.8 \mathrm{~Hz}, 2 \mathrm{H}, \mathrm{Ar}-\mathrm{H}), 7.31-7.23(\mathrm{~m}, 2 \mathrm{H}, \mathrm{Ar}-\mathrm{H}$, $\mathrm{CH}=\mathrm{CH}-\mathrm{CO}$ ), 7.03-6.90 (m, 4H, Ar-H, Ar- $\mathrm{CH}=\mathrm{CH}-), 6.71$ $(\mathrm{d}, J=8.8 \mathrm{~Hz}, 2 \mathrm{H}, \mathrm{Ar}-\mathrm{H}), 6.26(\mathrm{~d}, J=14.9 \mathrm{~Hz}, 1 \mathrm{H}, \mathrm{CH}=\mathrm{CH}-$ CO), 6.06 (s, $\left.2 \mathrm{H},-\mathrm{OCH}_{2} \mathrm{O}-\right) .{ }^{13} \mathrm{C}$ NMR $\left(101 \mathrm{MHz}, \mathrm{DMSO}-\mathrm{d}_{6}\right)$ : $\delta 163.69,153.86,148.42,148.26,140.60,138.78,131.55,131.31$, 125.66, 125.26, 123.21, 121.31, 115.58, 108.91, 106.18, 101.74 . ESI: $m / z 310.1[\mathrm{M}+1]^{+}$.

The crude $\mathrm{H}-2$ product was then purified using column chromatography on a silica gel (mixture eluent: $\mathrm{EA}: \mathrm{PE}=2: 1$ ) to obtain a $102 \mathrm{mg}$ product (4a) (yield: $71 \%$ ) as a pale-yellow solid. ${ }^{1} \mathrm{H}$ NMR (400 MHz, DMSO-d 6 ): $\delta 9.96$ (s, $1 \mathrm{H}, \mathrm{Ar}-\mathrm{OH}$ ), 9.60 (s, $1 \mathrm{H},-\mathrm{CO}-\mathrm{NH}-), 7.77$ (d, $J=8.0 \mathrm{~Hz}, 1 \mathrm{H}, \operatorname{Ar}-H)$, 


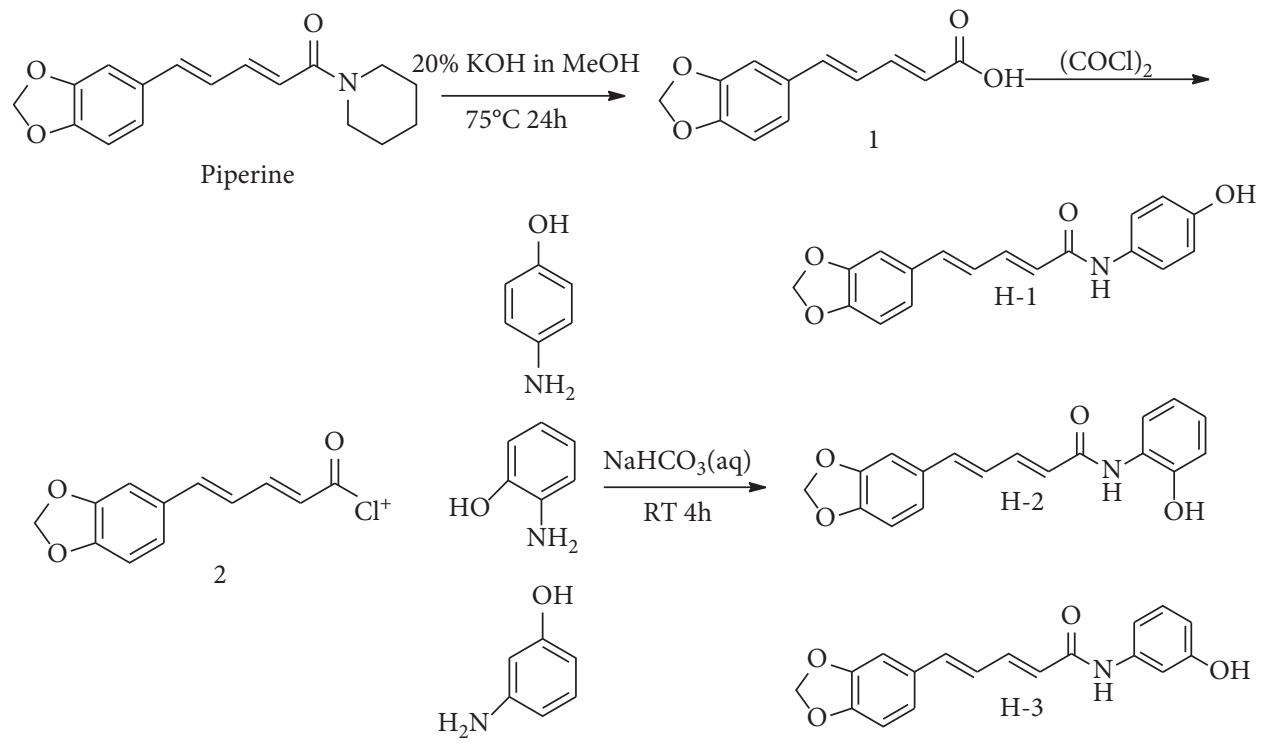

Figure 1: Schematic used in the preparation of piperine derivatives.

7.36-7.29 (m, 2H, Ar-H, CH=CH-CO), 7.04-6.87 (m, 6H, Ar$\mathrm{H}, \mathrm{Ar}-\mathrm{CH}=\mathrm{CH}-), 6.79$ (td, $J=7.6,1.6 \mathrm{~Hz}, \mathrm{Ar}-\mathrm{CH}=\mathrm{CH}-), 6.53$ (d, $J=14.9 \mathrm{~Hz}, \mathrm{CH}=\mathrm{CH}-\mathrm{CO}), 6.06\left(\mathrm{~s}, 2 \mathrm{H},-\mathrm{OCH}_{2} \mathrm{O}-\right),{ }^{13} \mathrm{C}$ NMR (101 MHz, DMSO-d $\mathrm{d}_{6}$ ): $\delta 164.95,148.51,148.44,148.40$, $141.73,139.52,131.20,126.97,125.48,125.39,124.42,123.37$, $122.80,119.55,116.72,108.94,106.24,101.78$. ESI: $m / z 310.1$ $[\mathrm{M}+1]^{+}$.

Finally, the H-3 crude product was purified using column chromatography on a silica gel (mixture eluent: PE: $\mathrm{AE}: \mathrm{HAc}=2: 1: 0.05$ ) to obtain a $95 \mathrm{mg}$ product (4a) (yield: $66 \%)$ as a pale-yellow solid. ${ }^{1} \mathrm{H}$ NMR $\left(400 \mathrm{MHz}\right.$, DMSO- $\left.\mathrm{d}_{6}\right)$ : $\delta 10.00(\mathrm{~s}, 1 \mathrm{H},-\mathrm{CO}-\mathrm{NH}-), 7.33-7.27(\mathrm{~m}, 3 \mathrm{H}, \mathrm{CH}=\mathrm{CH}-\mathrm{CO}$, Ar-H), 7.09-6.92 (m, 6H, Ar-H, Ar- $\mathrm{CH}=\mathrm{CH}-), 6.45$ (d, $J=7.4 \mathrm{~Hz}, 1 \mathrm{H}, \quad$ Ar-CH=CH-, $6.29(\mathrm{~d}, J=14.9 \mathrm{~Hz}, 1 \mathrm{H}$, $\mathrm{CH}=\mathrm{CH}-\mathrm{CO}), 6.06\left(\mathrm{~s}, 2 \mathrm{H},-\mathrm{OCH}_{2} \mathrm{O}-\right) .{ }^{13} \mathrm{C}$ NMR $(101 \mathrm{MHz}$, $\left.\mathrm{CDCl}_{3}\right): \delta 164.20,158.16,148.43,148.34,141.23,140.88$, $139.22,131.26,129.75,125.57,125.05,123.32,110.88,110.44$, 108.93, 106.87, 106.22, 101.76. ESI: $m / z 310.1[\mathrm{M}+1]^{+}$.

\subsection{In Vitro Antioxidant Activity Assessment of Target Compounds}

2.3.1. DPPH Assay. DPPH (2, 2-diphenyl-1-picrylhydrazyl) is regarded as a generator of free radicals and is widely used to quantify the antioxidative capacity of biological samples and foodstuff. In this study, a microplate reader was used to measure the in vitro antioxidative capacity of piperine and its derivatives applying the DPPH assay. DPPH free radical scavenging was tested according to the method reported by Gao et al. [16]. Piperine $(0.1 \mathrm{~mL})$ and its derivatives $(0.078125 \sim 5 \mathrm{mmol} / \mathrm{L})$ were added to $0.1 \mathrm{~mL}$ of a $0.1 \mathrm{mmol} / \mathrm{L}$ DPPH-ethanol solution. The reaction solution was incubated under darkened conditions at room temperature for 30 min. A microplate reader (Thermo Fisher, 1510) was used to measure absorbance $\left(D_{i}\right)$ at $517 \mathrm{~nm}$. An ethanol solution $(0.1 \mathrm{~mL})$ was separately mixed with $0.1 \mathrm{~mL}$ of the DPPHethanol solution or $0.1 \mathrm{~mL}$ of the sample solution, and absorbance $\left(D_{0}\right.$ and $\left.D_{j}\right)$ was then measured. $V_{\mathrm{C}}$ was used as the control. The experiment was carried out in parallel triplicate, and the following was used to calculate the DPPH free radical clearance rate (IP) and $\mathrm{IC}_{50}$ :

$$
\mathrm{IP}=\left(1-\frac{D_{i}-D_{j}}{D_{0}}\right) \times 100 \% .
$$

2.3.2. ABTS Assay. The ABTS (2, 2'-azino-bis(3-ethylbenzothiazoline-6-sulphonic acid) assay was conducted according to a modified method by Re et al. [17]. The ABTS working cation solution $\left(\mathrm{ABTS}^{+}\right)$was produced through reaction of the ABTS aqueous solution with potassium persulfate $\left(\mathrm{K}_{2} \mathrm{~S}_{2} \mathrm{O}_{8}\right)$ and kept under darkened conditions for $12 \sim 16 \mathrm{~h}$. The solution was then diluted to a concentration with an absorbance of $0.6 \sim 0.8$ at $734 \mathrm{~nm}$. Piperine $(0.1 \mathrm{~mL})$ and its derivatives $(1.25 \sim 80 \mu \mathrm{mol} / \mathrm{L})$ were added to $0.1 \mathrm{~mL}$ of the ABTS solution. The reaction solution was incubated under darkened conditions at room temperature for $30 \mathrm{~min}$. Absorbance $\left(D_{i}\right)$ was measured at $734 \mathrm{~nm}$. The ethanol solution $(0.1 \mathrm{~mL})$ was separately mixed with $0.1 \mathrm{~mL}$ of the ABTS solution or $0.1 \mathrm{~mL}$ of the sample solution, and the absorbance $\left(D_{0}\right.$ and $\left.D_{j}\right)$ was subsequently measured. $V_{\mathrm{C}}$ was used as a negative control. The experiment was carried out in parallel triplicate, and equation (1) was used to calculate the ABTS free radical clearance rate (IP) and $\mathrm{IC}_{50}$.

2.3.3. Protective Effects of Piperine Derivatives on Oxidatively Damaged Erythrocytes. Centrifugation (5 min at $3500 \mathrm{rpm} /$ min) was used to separate erythrocytes and plasma with the addition of an anticoagulant at $4^{\circ} \mathrm{C}$. The erythrocyte pellet was collected and washed four times with phosphate-buffered saline (PBS) $\left(\mathrm{pH} \mathrm{7.4)}\right.$ at $4^{\circ} \mathrm{C}$, followed by centrifugation at $3500 \mathrm{rpm} / \mathrm{min}$ for $5 \mathrm{~min}$. A suitable amount of PBS was then added to evenly mix and dilute the pellet to obtain a $10 \%$ erythrocyte suspension. 
The erythrocyte suspension $(100 \mu \mathrm{L})$ was added to the sample solution $(50 \mu \mathrm{L})$ and incubated at $37^{\circ} \mathrm{C}$ for $30 \mathrm{~min}$. Following this, $100 \mu \mathrm{L}$ of $200 \mathrm{mmol} / \mathrm{L} \mathrm{AAPH}$ was added, and the solution was incubated at $37^{\circ} \mathrm{C}$ for $1-4 \mathrm{~h}$ [18]. The erythrocyte reaction solution $(200 \mu \mathrm{L})$ was then aspirated, added to $1 \mathrm{~mL}$ of the PBS buffer, and centrifuged at $4^{\circ} \mathrm{C}$ and $3500 \mathrm{rpm} / \mathrm{min}$ for $5 \mathrm{~min}$. Following this, $200 \mu \mathrm{L}$ was added to a 96-well plate. The absorbance of the wells was measured at a $540 \mathrm{~nm}$ wavelength and recorded as $A_{\mathrm{PBS}}$. An identical volume of the erythrocyte reaction solution was aspirated and added to $1 \mathrm{~mL}$ of $4^{\circ} \mathrm{C}$ precooled double distilled water to complete erythrocyte lysis. Centrifugation was carried out under identical conditions, and the absorbance was measured at $540 \mathrm{~nm}$ and recorded as $A_{\text {water }}$. The experiment was carried out in parallel triplicate, and hemolysis rates were calculated using

$$
\text { Hemolysis rate }(\%)=\left(\frac{A_{\mathrm{PBS}}}{A_{\text {water }}}\right) \times 100 \% \text {. }
$$

The erythrocyte suspension $(100 \mu \mathrm{L})$ was added to the sample solution $(50 \mu \mathrm{L})$ and incubated at $37^{\circ} \mathrm{C}$ for $30 \mathrm{~min}$. Following this, $100 \mu \mathrm{L}$ of $200 \mathrm{mmol} / \mathrm{L}$ AAPH was added, and the oxidative damage treatment was carried out at $37^{\circ} \mathrm{C}$ for $1-4 \mathrm{~h}$. The erythrocyte reaction solution $(200 \mu \mathrm{L})$ was then aspirated, added to precooled double-distilled water $(100 \mu \mathrm{L})$, and centrifuged at $4^{\circ} \mathrm{C}$ and $3500 \mathrm{rpm}$ for $5 \mathrm{~min}$. The absorbance of the $200 \mu \mathrm{L}$ supernatant was measured at $630 \mathrm{~nm}$ and $700 \mathrm{~nm}$ wavelengths and recorded as $A_{630}$ and $A_{700}$, respectively. Following this, $10 \mu \mathrm{L}$ of a $5 \%$ potassium ferricyanide solution was added, and the absorbance was measured and recorded as $A_{\text {metHB630 }}$ and $A_{\text {metHB700, re- }}$ spectively. The hemoglobin oxidation rate was calculated using

Hemoglobin oxidation rate $(\%)=\left[\frac{\left(A_{630}-A_{700}\right)}{\left(A_{\text {metHB630 }}-A_{\text {metHB700 }}\right)}\right] \times 100 \%$.

\subsubsection{Effect of Piperine Derivatives on AAPH-Induced Rat} Erythrocyte Antioxidant Enzyme System and MDA Content. The erythrocyte suspension $(100 \mu \mathrm{L})$ was added to the sample solution $(50 \mu \mathrm{L})$ and incubated at $37^{\circ} \mathrm{C}$ for $30 \mathrm{~min}$. Following this, $100 \mu \mathrm{L}$ of $200 \mathrm{mmol} / \mathrm{L} \mathrm{AAPH}$ was added and the solution was incubated at $37^{\circ} \mathrm{C}$ for $1-4 \mathrm{~h}$. Precooled $\left(4^{\circ} \mathrm{C}\right)$ ultrapure water was added to the aforementioned erythrocyte reaction solution to complete erythrocyte lysis, and the solution was centrifuged. The pellet was collected and washed thrice with PBS. The erythrocyte pellet $(10 \mu \mathrm{L})$ from the previous wash was aspirated, added to $990 \mu \mathrm{L}$ of $4^{\circ} \mathrm{C}$ precooled double-distilled water, and stored in a freezer at $-80^{\circ} \mathrm{C}$ for subsequent experiments. SOD and MDA levels and GSH-Px and CAT activities in erythrocytes were measured following the manufacturer's instructions.

2.4. Data Processing. The experiments were carried out in parallel triplicate. All data was expressed as the mean\pm standard deviation $(\bar{x} \pm s)$. SPSS 22.0 software was used for statistical analysis of the data. One-way analysis of variance (one-way ANOVA) was used for data analysis. Finally, $p<0.05$ was considered significant.

\section{Results and Discussion}

3.1. DPPH and ABTS Free Radical Scavenging Ability of Piperine Derivatives. DPPH and ABTS are regarded as free radical generators and widely used to quantify antioxidative capacities of biological samples and foodstuff. In this study, a microplate reader was used to measure the in vitro antioxidative capacity of piperine and its derivatives using $\mathrm{DPPH}$ and ABTS free radical clearance assays.

As shown in Figure $2(\mathrm{a}), V_{\mathrm{C}}$ was used as a positive control, and its ability to scavenge $\mathrm{DPPH}^{\bullet}$ free radicals was the highest overall. Piperine had almost no scavenging effect on $\mathrm{DPPH}^{\bullet}$ free radicals. After its structural modification, the ability of piperine to scavenge $\mathrm{DPPH}^{\bullet}$ free radicals increased and exhibited concentration dependence. Under identical concentrations, the $\mathrm{DPPH}^{\bullet}$ free radical clearance rate of piperine derivative 1 was higher than piperine derivatives 2 and 3 . Greater than $80 \%$ of $\mathrm{DPPH}^{\bullet}$ free radicals were cleared at $0.625 \mathrm{mmol} / \mathrm{L}$ of piperine derivative 1 . The $\mathrm{IC}_{50}$ value reflects the $50 \%$ inhibitory concentration of the antioxidant being measured. Therefore, lower $\mathrm{IC}_{50}$ values correspond to the higher antioxidative capacity of the substance. As shown in Table 1, the antioxidative capacity of the different derivatives was as follows: $V_{\mathrm{C}}>\mathrm{H}-1>\mathrm{H}-2>\mathrm{H}-3>$ piperine.

Figure 2(b) provides ABTS free radical clearance results for piperine and its derivatives. Piperine did not exhibit any clearance of ABTS free radicals. After structural modification, however, the ability of piperine to clear ABTS free radicals increased, and the clearance rate increased as concentrations increased. At a $40 \mu \mathrm{mol} / \mathrm{L}$ concentration, ABTS free radical clearance rates of $\mathrm{H}-1, \mathrm{H}-2$, and $\mathrm{H}-3$ were all above $90 \%$. After $\mathrm{IC}_{50}$ calculations, the antioxidative capacity of the different derivatives was as follows: $\mathrm{H}-3>\mathrm{H}$ $2>\mathrm{H}-1>V_{\mathrm{C}}>$ piperine. However, there were no significant statistical differences detected in the $\mathrm{IC}_{50}$ of $\mathrm{H}-2$ and $\mathrm{H}-3$.

3.2. Protective Effects of Piperine Derivatives on AAPH-Induced Erythrocyte Oxidative Hemolysis and Erythrocyte Hemoglobin Oxidation. AAPH is the most common free radical inducer used in the construction of erythrocyte oxidative damage models. Studies have shown that AAPH has a strong ability to induce oxidative damage in erythrocytes, resulting in hemolysis. For this study, we constructed an erythrocyte oxidative damage model. Firstly, we assessed the protective abilities of piperine and its derivatives on AAPH-induced erythrocyte oxidative hemolysis, while further examining the effects of piperine derivatives on hemoglobin oxidation rates in erythrocytes. Erythrocytes are the best cellular model to study oxidative damage $[19,20]$. Experimental results showed that, at a $200 \mathrm{mmol} / \mathrm{L} \mathrm{AAPH}$ concentration and incubation at $37^{\circ} \mathrm{C}$ for $2 \mathrm{~h}$, the erythrocyte hemolysis rate was $>60 \%$, comprising of greater than half the number of erythrocytes. Moreover, no significant differences were observed between $2 \mathrm{~h}$ and $4 \mathrm{~h}$ of incubation. Therefore, 


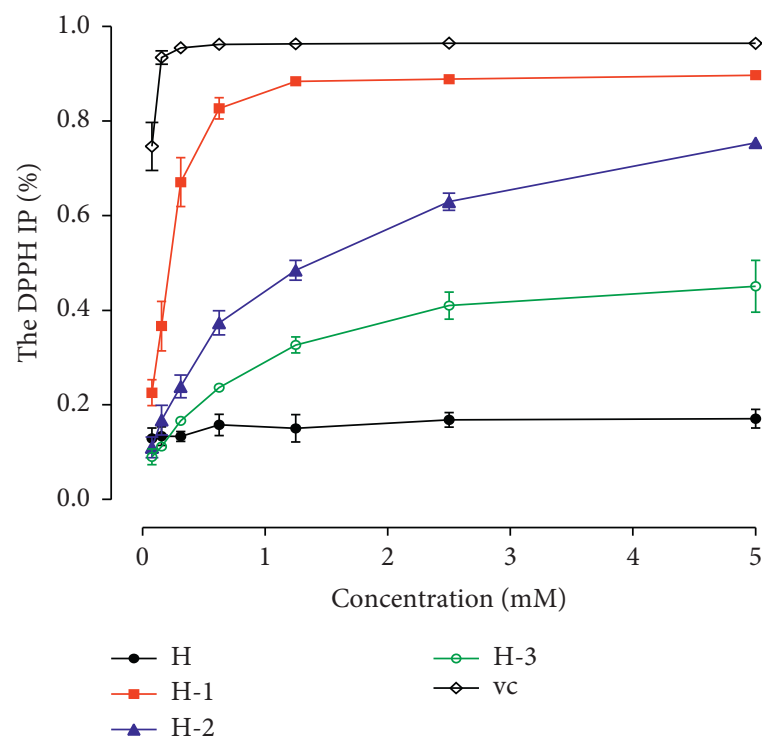

(a)

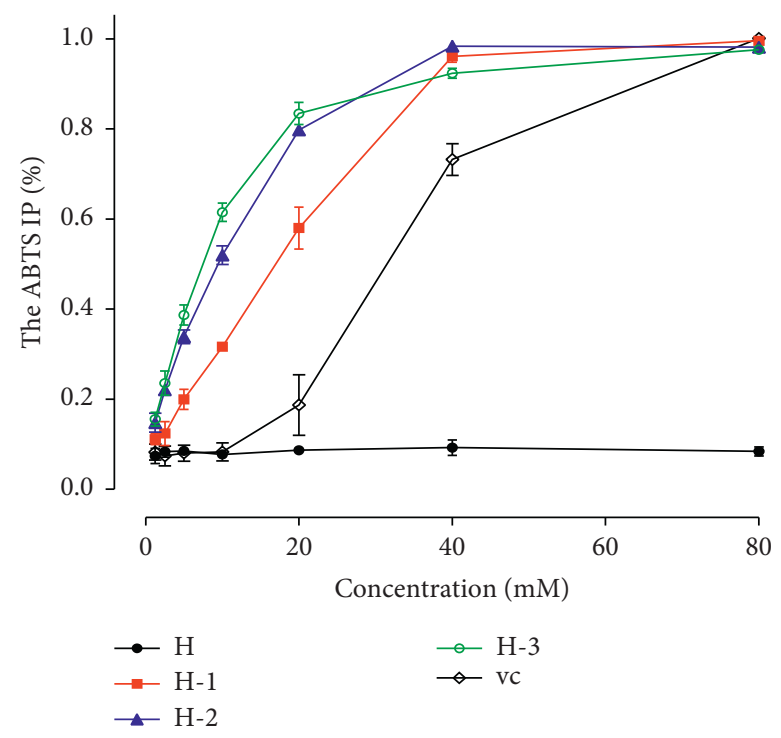

(b)

Figure 2: DPPH and ABTS free radical clearance curves of piperine and its derivatives. (a) The DPPH free radical clearance rate (IP) of piperine and its derivatives at different concentrations. (b) The ABTS free radical clearance rate (IP) of piperine and its derivatives at different concentrations.

TABLE 1: $\mathrm{IC}_{50}$ of the DPPH and ABTS free radical inhibition rates of the different piperine derivatives.

\begin{tabular}{lcc}
\hline Compound & $\mathrm{IC}_{50}$ of DPPH radical oxidation inhibition rate $(\mathrm{mM})$ & $\mathrm{IC}_{50}$ of ABTS radical oxidation inhibition rate $(\mu \mathrm{M})$ \\
\hline Piperine & - & - \\
$\mathrm{H}-1$ & $0.21 \pm 0.43$ & $8.70 \pm 0.092$ \\
$\mathrm{H}-2$ & $1.25 \pm 0.14$ & $6.15 \pm 0.066$ \\
$\mathrm{H}-3$ & $7.82 \pm 2.52$ & $6.04 \pm 0.34$ \\
$V_{\mathrm{C}}$ & $0.00097 \pm 0.00078$ & $9.80 \pm 1.46$ \\
\hline
\end{tabular}

incubation at $37^{\circ} \mathrm{C}$ for $2 \mathrm{~h}$ was selected for our subsequent experiments.

As shown in Figure 3(a), erythrocytes were stable after $1-4 \mathrm{~h}$ of incubation in PBS (the control group). After $1 \mathrm{~h}$ of AAPH oxidative stress, no hemolysis occurred. Subsequently, as incubation time increased, the hemolysis rate increased significantly, reaching a maximum hemolysis rate at $2 \mathrm{~h}$. When erythrocytes were preincubated with piperine derivatives prior to $2 \mathrm{~h}$ of AAPH stress, their hemolysis rates decreased compared to the AAPH model group $(p<0.05)$. Moreover, damage to hemolysis rates after $2 \mathrm{~h}$ of AAPH stress showed that percentages of $\mathrm{H}, \mathrm{H}-1, \mathrm{H}-2$, and $\mathrm{H}-3$ were $72.60 \%, 66.84 \%, 49.23 \%, 52.08 \%$, and $64.07 \%$, respectively. This confirmed that the compounds exhibited protective effects towards AAPH-induced erythrocyte lysis and that $\mathrm{H}-1$ and $\mathrm{H}-2$ activities were higher compared to $\mathrm{H}-0$. Figure 3(b) provides concentration effects of samples while being protected against AAPH-induced erythrocyte lysis. Results showed that when the hemolysis rates of $\mathrm{H}-1, \mathrm{H}-2$, and $\mathrm{H}-3$ were compared to piperine, $\mathrm{H}-1$ had a significant hemolysis rate reduction $(p<0.05)$. The protective ability of $\mathrm{H}-1$ towards AAPH-induced erythrocyte lysis also exhibited concentration dependence; namely, when concentrations were 25,50 , and $100 \mu \mathrm{mol} / \mathrm{L}, \mathrm{H}-1$ hemolysis rates were $59.42 \%, 49.23 \%$, and $20.0 \%$, respectively. These results show that piperine and its derivatives exhibit protective effects against AAPH-induced erythrocyte oxidative hemolysis, wherein the protective ability of $\mathrm{H}-1$ was stronger compared with the parent compound.

Erythrocyte oxidative damage causes hemoglobin oxidation and formation of methemoglobin. Therefore, quantifying methemoglobin levels in erythrocytes can reflect the level of erythrocyte oxidative damage. As shown in Figure 4(a), methemoglobin levels in the control group were extremely low and stable. In the AAPH group, however, methemoglobin levels significantly increased as incubation time increased. This showed that methemoglobin levels increased in cells after AAPH damage to erythrocytes. After erythrocytes were preincubated with piperine derivatives followed by $1 \mathrm{~h}$ and $2 \mathrm{~h}$ of stress, piperine derivatives were found to be ineffective in reducing increases in AAPH-induced methemoglobin. However, after $4 \mathrm{~h}$ of stress, the $\mathrm{H}-1$ derivative significantly reduced AAPH-induced methemoglobin levels $(p<0.05)$. This showed that the $\mathrm{H}-1$ derivative has protective effects against AAPH-induced hemoglobin oxidation. Figure 4(b) shows concentration effects of piperine derivatives on AAPH-induced erythrocyte hemoglobin oxidation. Results showed that $\mathrm{H}-1$ exhibited protective effects against hemoglobin oxidation at 50 and $100 \mu \mathrm{mol} / \mathrm{L}$ concentrations. These findings showed that the $\mathrm{H}-1$ 


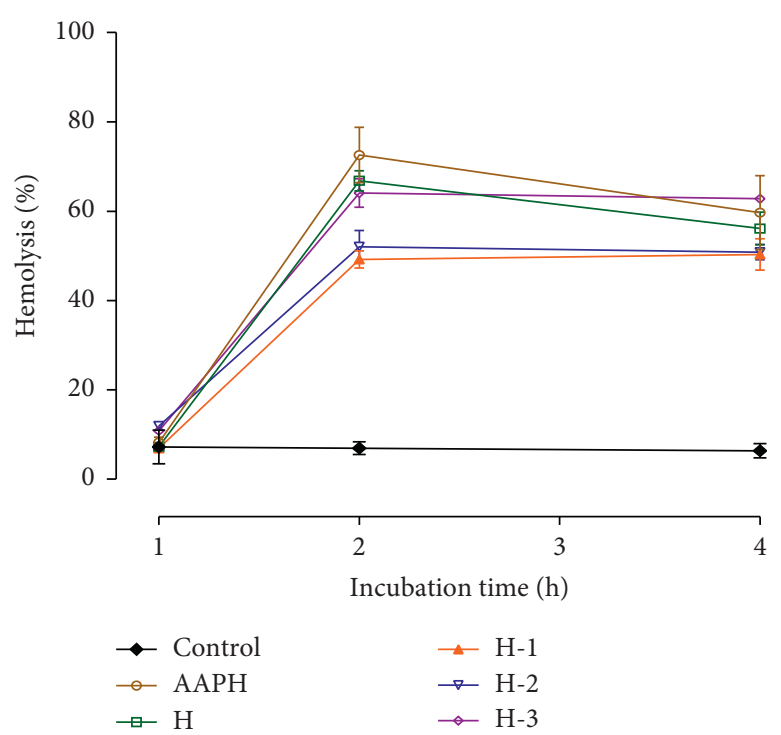

(a)

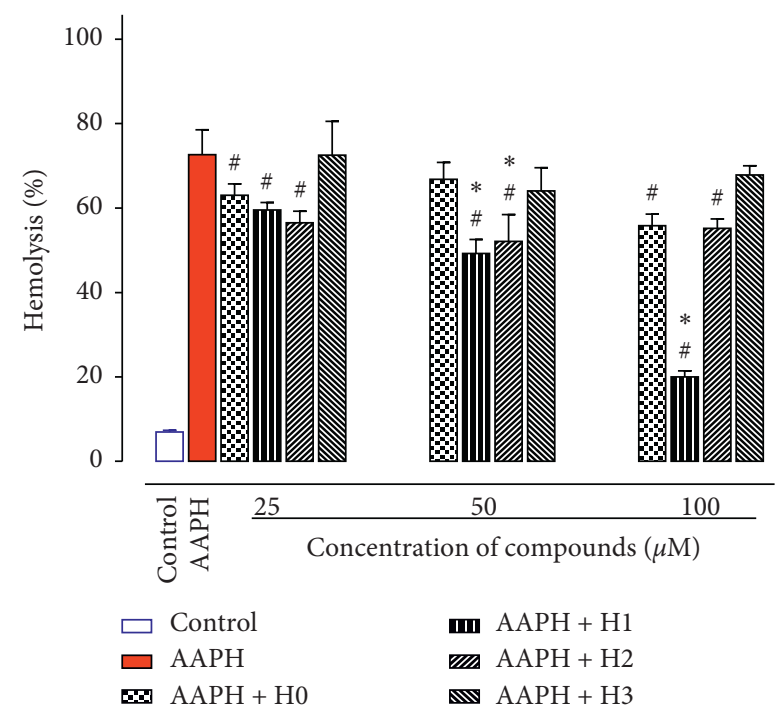

(b)

FIGURE 3: Protective effect duration of piperine derivatives on AAPH-induced erythrocyte hemolysis and time curves. (a) Time curves of the protective effects of piperine derivatives on AAPH-induced erythrocyte hemolysis at a concentration of $50 \mu \mathrm{M}$. (b) The relationship between the protective effects of piperine derivatives at different concentrations and AAPH-induced erythrocyte hemolysis after $2 \mathrm{~h}$ incubation.

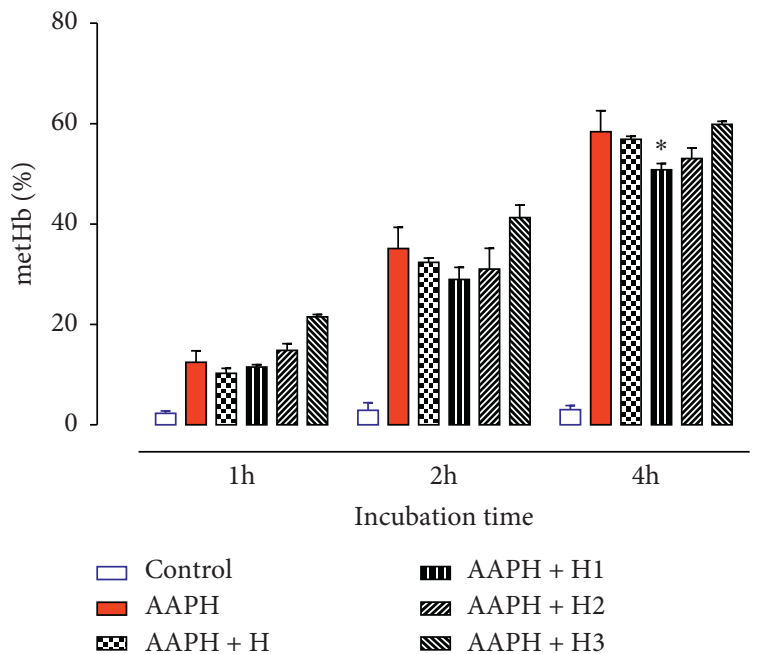

(a)

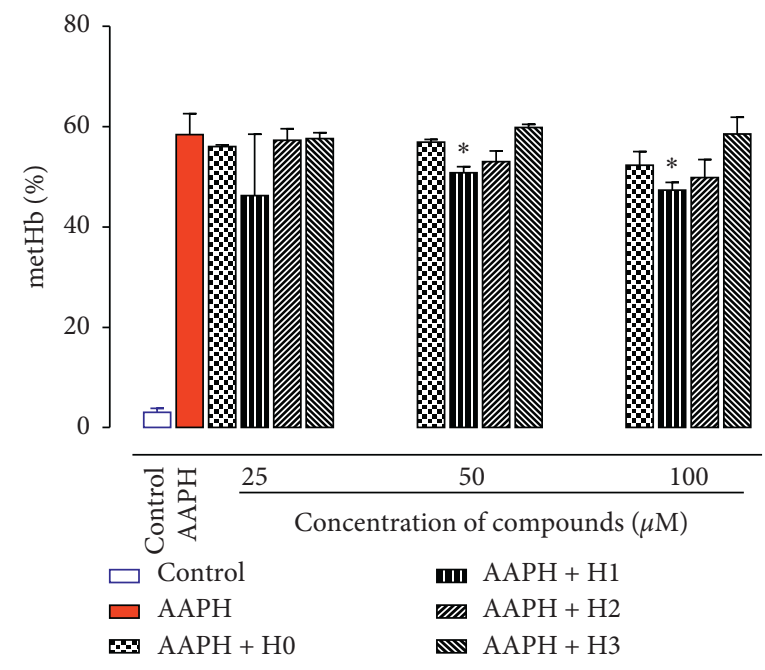

(b)

FIgURE 4: Duration of the protective effects of piperine derivatives on AAPH-induced hemoglobin oxidation in erythrocytes and time curves. (a) Time curves of the protective effects of piperine derivatives on AAPH-induced hemoglobin oxidation at a concentration of $50 \mu \mathrm{M}$. (b) The relationship between the protective effects of piperine derivatives at different concentrations and AAPH-induced hemoglobin oxidation after $4 \mathrm{~h}$ incubation.

derivative had protective effects against AAPH-induced hemoglobin oxidation in erythrocytes.

\subsection{Effects of Piperine Derivatives on AAPH-Induced Eryth-} rocyte GSH-Px, SOD, and CAT Activity. Free radicals (i.e., ROS and reactive nitrogen species (RNS)) possess strong chemical reactivity and have the potential to cause oxidative damage to biological macromolecules. Under normal circumstances, oxidative and antioxidative systems in the body maintain a certain balance to protect tissues and organs from being attacked by oxidizing agents. Among the body's enzymatic and nonenzymatic antioxidative systems, superoxide dismutase (SOD), catalase (CAT), and glutathione peroxidase (GSH-Px) are major antioxidant enzyme systems $[21,22]$. GSH-Px is an antioxidant enzyme that is ubiquitous in different tissues and cells in the human body. GSH-Px can reduce glutathione $(\mathrm{GSH})$ and generate hydrogen peroxide $\left(\mathrm{H}_{2} \mathrm{O}_{2}\right)$ inside cells to scavenge peroxides in the body as well as also having antilipid oxidation effects. SOD is a crucial 


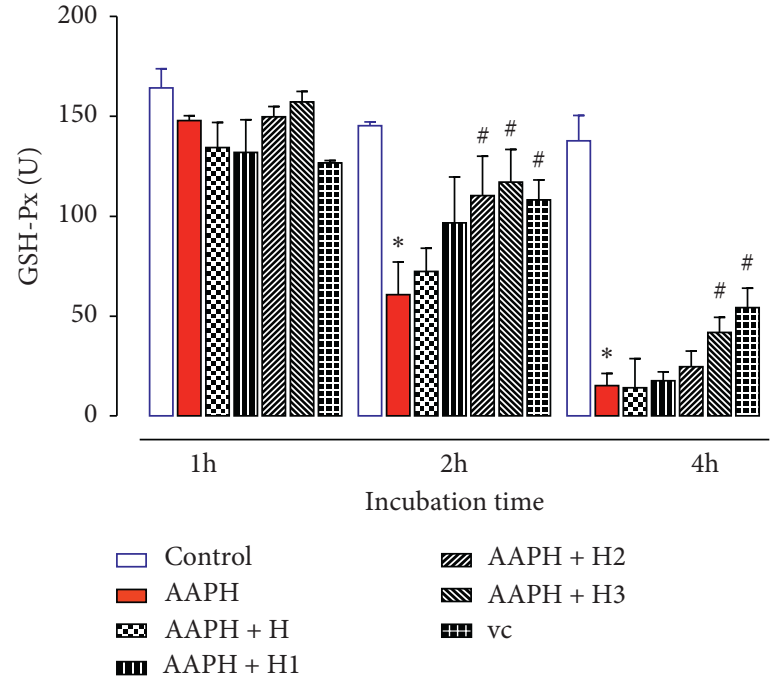

(a)

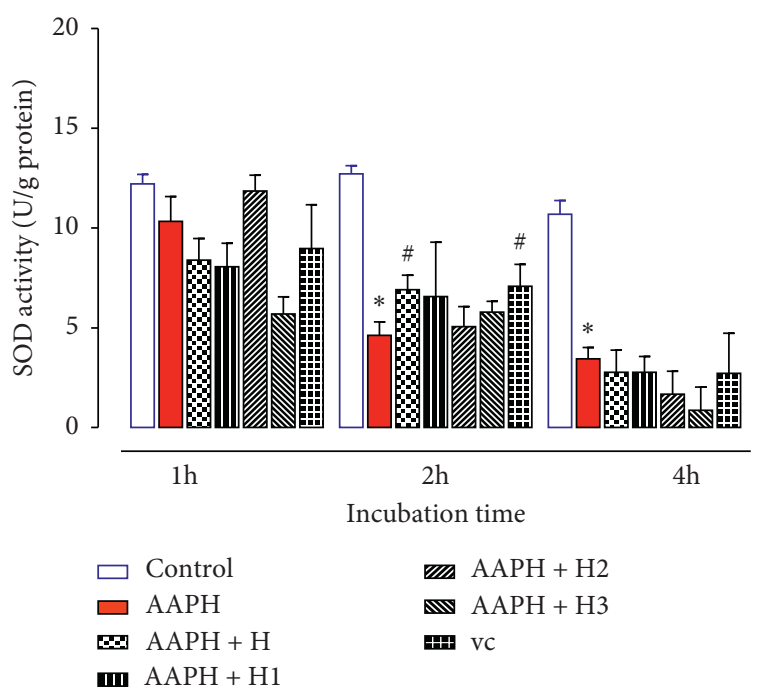

(b)

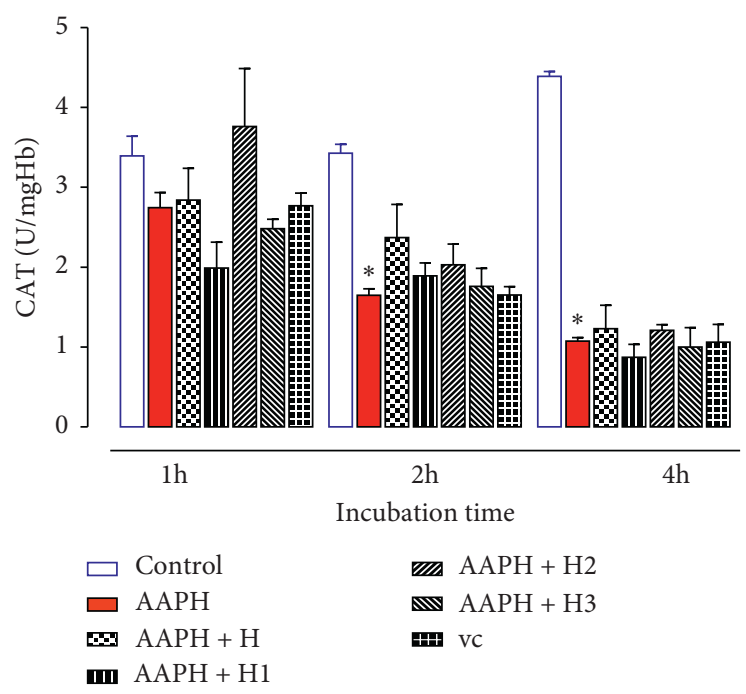

(c)

FIGURE 5: Effects of piperine derivatives on AAPH-induced GSH-Px levels, SOD activity, and CAT activity in rat erythrocytes.

enzyme that scavenges free radicals in the body, and its activity can reflect the ability of the body to scavenge free radicals. CAT, ubiquitous in erythrocytes, catalyzes $\mathrm{H}_{2} \mathrm{O}_{2}$ degradation into $\mathrm{H}_{2} \mathrm{O}$ and $\mathrm{O}_{2}$, thereby reducing the oxidative damage caused by the oxidation product $\mathrm{OH}^{\bullet}$ produced by $\mathrm{H}_{2} \mathrm{O}_{2}$ and metal ions [23].

Figure 5(a) shows the effects of piperine derivatives on AAPH-induced GSH-Px activity in erythrocytes. In the control group (i.e., the non-AAPH stress group), GSH-Px levels in erythrocytes were stable. In the AAPH stress group, GSH-Px levels in erythrocytes gradually decreased as incubation time increased. After 1, 2, and $4 \mathrm{~h}$ of AAPH stress, GSH-Px levels in erythrocytes successively decreased, and the differences were statistically significant.

When $50 \mu \mathrm{M}$ of piperine derivatives were added to erythrocytes prior to AAPH oxidative stress and compared to the AAPH group, no significant effects on GSH-Px levels were observed in erythrocytes after $1 \mathrm{~h}$. At $2 \mathrm{~h}, \mathrm{H}-2$ and $\mathrm{H}-3$ significantly increased GSH-Px levels $(p<0.05)$. After $4 \mathrm{~h}$, only the H-3 group showed significant differences in GSHPx levels compared to the AAPH group $(p<0.05)$. These results showed that both $\mathrm{H}-2$ and $\mathrm{H}-3$ can increase GSH-Px activity in erythrocytes, and between the two, the effect of $\mathrm{H}-3$ was the most significant while not exhibiting any significant differences compared to the positive control (i.e., $\left.V_{\mathrm{C}}\right)(p>0.05)$.

Figure 5(b) shows the effect of piperine derivatives on AAPH-induced SOD activity in erythrocytes. Compared to the control group, SOD activity in erythrocytes under the AAPH stress group gradually decreased with increasing incubation time. $50 \mu \mathrm{M}$ of piperine derivatives were added to erythrocytes prior to AAPH oxidative stress and compared with the AAPH group. No significant effects respective to SOD activity were observed in erythrocytes after $1 \mathrm{~h}$ of reaction time $(p>0.05)$. However, at $2 \mathrm{~h}, \mathrm{H}, \mathrm{H}-1$, and $\mathrm{H}-3$ significantly increased SOD activity $(p<0.05)$. After $4 \mathrm{~h}$, 


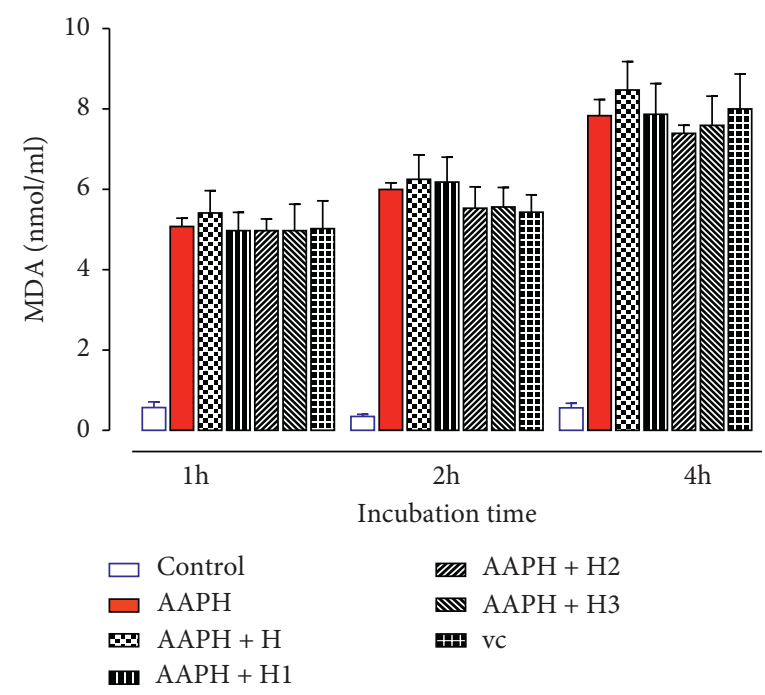

FIGURE 6: Effects of piperine derivatives on AAPH-induced MDA levels in rat erythrocytes.

piperine derivatives and the positive control (i.e., $V_{\mathrm{C}}$ ) exhibited no significant effects on SOD activity in erythrocytes $(p>0.05)$. These results showed that as the duration of stress increased to $2 \mathrm{~h}$, piperine and $V_{\mathrm{C}}$ increased SOD activity in erythrocytes $(p<0.05)$; however, the derivatives were unable to do so $(p<0.05)$. When the stress duration increased to $4 \mathrm{~h}$, no increase in SOD activity was observed in erythrocytes in any group compared with the AAPH group. When oxidative damage levels were severe, piperine was unable to effectively prevent SOD consumption in erythrocytes. These results showed that piperine derivatives exhibit no significant effects on SOD activity in AAPHdamaged erythrocytes.

Figure 5(c) shows the effects of piperine derivatives on AAPH-induced CAT activity in erythrocytes. Compared with the control group, CAT activity in erythrocytes from the AAPH stress group gradually decreased with increasing incubation time. After $50 \mu \mathrm{M}$ of piperine derivatives were added, piperine and its derivatives did not show any significant difference in intracellular CAT levels compared with the AAPH group $(p>0.05)$. These results showed that piperine and its derivatives do not cause any significant differences in AAPH-induced CAT activity in rat erythrocytes $(p>0.05)$.

3.4. Effects of Piperine Derivatives on AAPH-Induced MDA Levels in Rat Erythrocytes. A free radical imbalance in the body causes unsaturated fatty acid oxidation to occur, subsequently resulting in the formation of lipid peroxides, of which malondialdehyde (MDA) is the most classic reaction end-product. MDA causes cytotoxicity as it can disrupt the integrity of the phospholipid membrane and induce cell death [24]. MDA levels can reflect the level of tissue and cell damage. Compared to the control group, MDA levels in the AAPH group significantly increased as incubation time increased (Figure 6). This showed that AAPH induces ROS generation, which causes lipid peroxidation and the upregulation of MDA levels in cells. There were no significant differences in MDA levels in erythrocytes treated with piperine and its derivatives compared with the AAPH group $(p>0.05)$. These results showed that piperine and its derivatives do not protect against AAPH-induced lipid peroxidation.

\section{Conclusions}

In this study, naturally extracted piperine was used as a raw material to synthesize piperine derivatives containing a phenolic hydroxyl group. We demonstrated that the free radical clearance rates of piperine derivatives were higher compared with the parent compound. Piperine derivatives exhibited a potent antioxidant ability in vitro. By constructing an AAPH-induced erythrocyte oxidative damage model, we demonstrated that piperine derivatives have protective effects on AAPH-mediated oxidatively damaged erythrocytes. The ability of the $\mathrm{H}-1$ derivative to inhibit AAPH-induced erythrocyte lysis and hemoglobin oxidation was higher compared to the parent compound. We further investigated whether the protective effects of piperine derivatives against AAPH-induced oxidative damage may derive from the protective activity of the intracellular antioxidant enzyme system. Our results showed that piperine derivatives can increase GSH-Px activity in erythrocytes. In particular, the protective effect of the $\mathrm{H}-3$ derivative was shown to be better than piperine. To summarize, we designed and synthesized three piperine derivatives, which effectively increased the antioxidative capacity of piperine. This study provides a valuable research foundation for the application of piperine as an antioxidant.

\section{Data Availability}

The data used to support the findings of this study are available from the corresponding author upon request. 


\section{Conflicts of Interest}

The authors declare no conflicts of interest regarding the writing and publication of this study.

\section{Acknowledgments}

This study was financially supported by scientific research funds provided by Xi' an Medical University (2016YXXK09), Xi'an and Weiyang District Science and Technology Fund (201930), and Xi'an Science and Technology Bureau Project (2020KJRC0135).

\section{References}

[1] J. P. Kehrer and L.-O. Klotz, "Free radicals and related reactive species as mediators of tissue injury and disease: implications for Health," Critical Reviews in Toxicology, vol. 45, no. 9, pp. 765-798, 2015.

[2] A. Phaniendra, D. B. Jestadi, and L. Periyasamy, "Free radicals: properties, sources, targets, and their implication in various diseases," Indian Journal of Clinical Biochemistry, vol. 30, no. 1, pp. 11-26, 2015.

[3] K. Bagchi and S. Puri, "Free radicals and antioxidants in health and disease," Eastern Mediterranean Health Journal, vol. 4, pp. 350-360, 2008.

[4] A. Singh, P. Pandey, M. Tewari et al., "Free radicals hasten head and neck cancer risk: a study of total oxidant, total antioxidant, DNA damage, and histological grade," Journal of Postgraduate Medicine, vol. 62, pp. 96-101, 2016.

[5] E. S. V. Glinter and V. Panakova, "Antioxidants in health and disease," Bratislava Medical Journal, vol. 115, no. 10, pp. 603-606, 2014.

[6] L. J. Machlin and A. Bendich, "Free radical tissue damage: protective role of antioxidant nutrients 1," The FASEB Journal, vol. 1, no. 6, pp. 441-445, 1987.

[7] N. Verma, S. Bal, R. Gupta, and A. Yadav, "Antioxidative effects of piperine against cadmium-induced oxidative stress in cultured human peripheral blood lymphocytes," Journal of Dietary Supplements, vol. 17, no. 1, pp. 41-52, 2018.

[8] A. C. P. Reddy and B. R. Lokesh, "Studies on spice principles as antioxidants in the inhibition of lipid peroxidation of rat liver microsomes," Molecular and Cellular Biochemistry, vol. 111, no. 1-2, pp. 117-124, 1992.

[9] E. S. Sunila and G. Kuttan, "Immunomodulatory and antitumor activity of Piper longum Linn. and piperine," Journal of Ethnopharmacology, vol. 2, no. 3, pp. 339-346, 2004.

[10] W. S. Chen, J. An, J. J. Li et al., "Piperine attenuates lipopolysaccharide (LPS)-induced inflammatory responses in BV2 microglia," International Immunopharmacology, vol. 42, pp. 44-48, 2017.

[11] A. Khajuria, N. Thusu, U. Zutshi, and K. L. Bedi, "Piperine modulation of carcinogen induced oxidative stress in intestinal mucosa," Molecular and Cellular Biochemistry, vol. 189, no. 1-2, pp. 113-118, 1998.

[12] T. Velpandian, R. Jasuja, R. K. Bhardwaj, J. Jaiswal, and S. K. Gupta, "Piperine in food: interference in the pharmacokinetics of phenytoin," European Journal of Drug Metabolism and Pharmacokinetics, vol. 26, no. 4, pp. 241-247, 2001.

[13] J. P. Adjimani and P. Asare, "Antioxidant and free radical scavenging activity of iron chelators," Toxicology Reports, vol. 2, pp. 721-728, 2015.
[14] H. Matsuda, K. Ninomiya, T. Morikawa, D. Yasuda, I. Yamaguchi, and M. Yoshikawa, "Hepatoprotective amide constituents from the fruit of Piper chaba: structural requirements, mode of action, and new amides," Bioorganic \& Medicinal Chemistry, vol. 17, no. 20, pp. 7313-7323, 2009.

[15] C. Kharbanda, M. S. Alam, H. Hamid et al., "Novel piperine derivatives with antidiabetic effect as PPAR- $\gamma$ agonists," Chemical Biology \& Drug Design, vol. 88, no. 3, pp. 354-362, 2016.

[16] Y. T. Gao, W. Wei, L. Q. Ye et al., "Photometric micro-titration model of DPPH radicals scavenging activity and its application," Spectroscopy and Spectral Analysis, vol. 35, no. 2, pp. 492-496, 2015.

[17] R. Re, N. Pellegrini, A. Proteggente, A. Pannala, M. Yang, and C. Rice-Evans, "Antioxidant activity applying an improved ABTS radical cation decolorization assay," Free Radical Biology and Medicine, vol. 26, no. 9-10, pp. 1231-1237, 1999.

[18] L. Zheng, H. Dong, G. Su, Q. Zhao, and M. Zhao, "Radical scavenging activities of Tyr-, Trp-, Cys- and Met-Gly and their protective effects against AAPH-induced oxidative damage in human erythrocytes," Food Chemistry, vol. 197, pp. 807-813, 2016.

[19] K. A. Arbos, L. M. Claro, L. Borges, C. A. M. Santos, and A. M. Weffort-Santos, "Human erythrocytes as a system for evaluating the antioxidant capacity of vegetable extracts," Nutrition Research, vol. 28, no. 7, pp. 457-463, 2008.

[20] W. Liao, Z. Ning, L. Chen et al., "Intracellular antioxidant detoxifying effects of diosmetin on 2, 2-azobis(2-amidinopropane) dihydrochloride (AAPH)-induced oxidative stress through inhibition of reactive oxygen species generation," Journal of Agricultural and Food Chemistry, vol. 62, no. 34, pp. 8648-8654, 2014.

[21] J. Kim, A. Mizokami, M. Shin et al., "SOD3 acts as a tumor suppressor in PC-3 prostate cancer cells via hydrogen peroxide accumulation," Anticancer Research, vol. 6, pp. 28212831, 2014.

[22] H. Nishikawa and S. Kitani, "Tea catechins have dual effect on mast cell degranulation induced by compound 48/80," International Immunopharmacology, vol. 8, no. 9, pp. 12071215, 2008.

[23] M. Y. B. Çimen, "Free radical metabolism in human erythrocytes," Clinica Chimica Acta, vol. 390, no. 1-2, pp. 1-11, 2008.

[24] S. Luqman and S. I. Rizvi, "Protection of lipid peroxidation and carbonyl formation in proteins by capsaicin in human erythrocytes subjected to oxidative stress," Phytotherapy Research, vol. 20, no. 4, pp. 303-306, 2006. 